\title{
Using model-based predictions to inform the mathematical aggregation of human-based predictions of replicability
}

Working Draft Manuscript

AUTHORS

Elliot Gould

David Wilkinson

Rebecca Groenewegen

Felix Singleton-Thorn

Hannah Fraser

Aaron Willcox

Peter Vesk
AFFILIATIONS

School of Ecosystem and Forest Sciences, University of Melbourne

School of Ecosystem and Forest Sciences, University of Melbourne

School of Ecosystem and Forest Sciences, University of Melbourne

School of Historical and Philosophical Studies, University of Melbourne

School of Historical and Philosophical Studies, University of Melbourne

School of Historical and Philosophical Studies, University of Melbourne

School of Ecosystem and Forest Sciences, University of Melbourne

PUBLISHED

May 1, 2021

\section{Introduction}

Eight recent large scale replication studies have been conducted to examine the reproducibility and heterogeneity of effect sizes in psychological research. These projects involved hundreds of researchers, tens of thousands of participants, and now provide a resource of over 300 individual replication studies.

An essential question in assessing the published literature is what are the markers of replication likelihood. Should we believe that a "successful" replication is more likely if the original study has a large sample size? Should we think it less likely if the effect of interest is an interaction effect? This large database of replication studies gives us an opportunity to begin to answer these questions. 
Crowd-sourced human judgments about the trustworthiness of research claims may be used to generate forecasts of the probability of those claims being successfully replicated . Predictive models are less time and resource-intensive methods of generating forecasts about the likely replicability of research claims , however, are likely to be less accurate than human judgments. In this paper we propose and demonstrate a method that combines both crowd-sourced human judgments and model-based predictions of the likely replicability of research claims. We employ a Bayesian model that takes the model-based predictions as the prior data, and updates these values using crowd-sourced human judgments to generate predictions of replication outcomes using an annotated database of eight large-scale replication studies.

\section{Methods}

\section{Dataset Creation}

A number of data sources were pulled together to prepare this database. Firstly, each of the large scale replication projects' data sets was downloaded or extracted from tables. Secondly, the DOIs for each original study were extracted and used to download all available data from Web of Science. Thirdly, PDFs for each available article were downloaded and a team of $13 \mathrm{PhD}$ students and postdocs manually coded each of the original studies. Finally, the PDFs were converted to plain text and the program statcheck was run on each of the original studies. Full details about each of these data sourcing efforts are reported below.

\section{Data sourced from replication projects}

All eight published or in-press large scale replication projects performed within in the behavioral science research literature were included in the current study (see Table 1 for a list of the included studies), the data were originally used in [FST replication ES study] and this description is lightly adapted from that paper. Each paper had different sampling frames that are described below.

The original source of each replicated effect, reported test statistics, effect sizes, sample sizes, standard errors and $p$-values were extracted for each original and replication study. Several of the large scale replication projects did not present the original test statistics and $p$ values (e.g., Many Labs 1 and 3, Ebersole et al., 2016; Klein et al., 2014). In these cases, these values were manually extracted from the original articles. When sample sizes for original studies were not reported in the data provided by each replication project they were manually extracted from original articles where possible. For all analyses, the original and replication effect sizes were transformed to Fisher ztransformed correlation coefficients following the methods used in Open Science Collaboration (2015, see Supplementary Materials 2 for details). This conversion used data from the replication project whenever possible (i.e., whenever effect sizes were reported in correlation coefficients in a summary table or in a project's online data this was directly converted to Fisher $z$ values). If the study-level 
the original or replication project we excluded the result from this analysis (e.g., cases when no effect size was reported in the original study or in the replication project data set).

In cases where sample sizes were not reported per group, sample sizes among groups were assumed to be equal in these conversions. For each of the Many Labs projects the top level result was used (i.e., the results of the analysis that collapsed the data across the multiple labs) because of the difficulty of extracting results at the study level. See Supplementary Materials 1 for a comprehensive account of exclusions and study specific extraction details for each replication project.

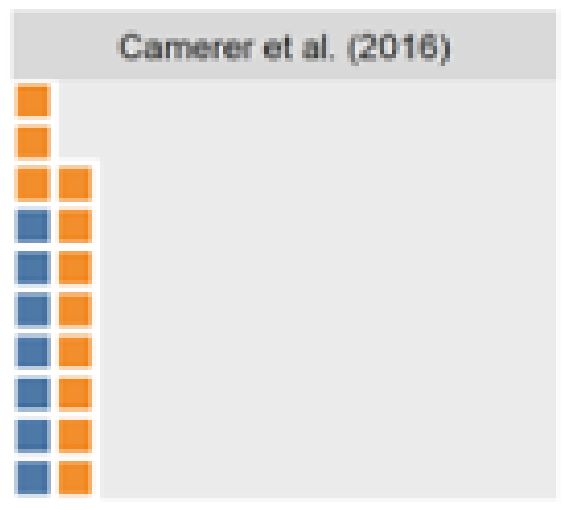

Ebersole ot al, (2016)

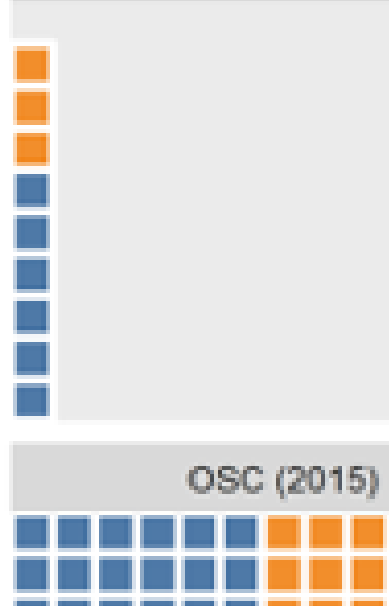

Camerer, et al, (2018)
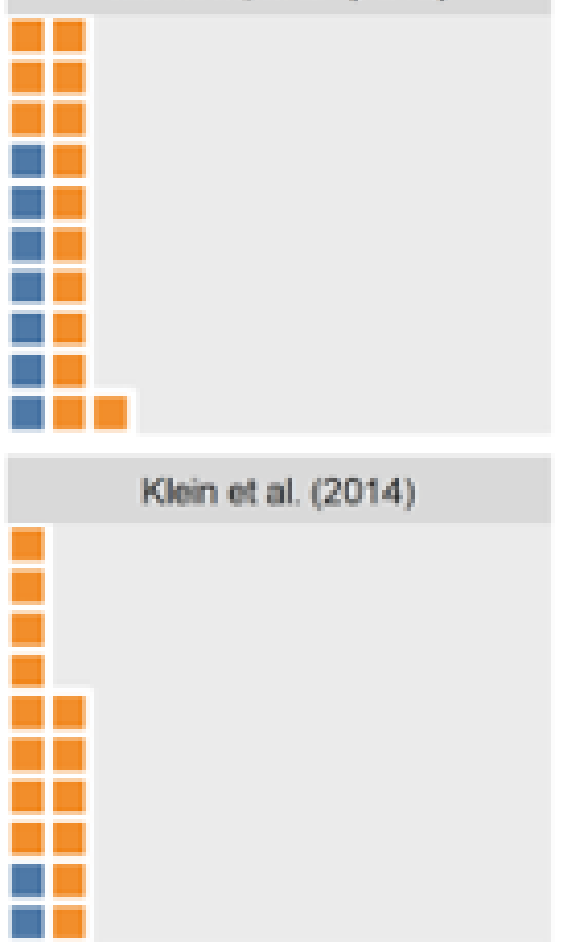

Klein ef al. (2014)

Solo (2019)

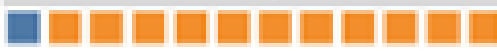
Q
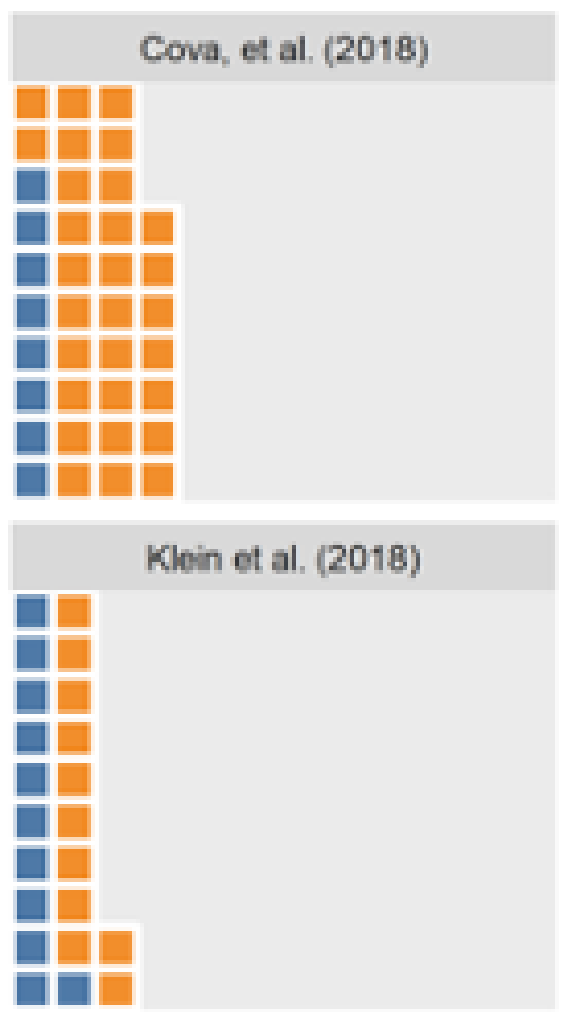

Replication significant in the oriainal direction 


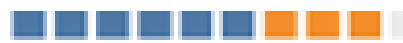

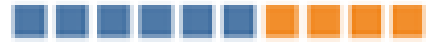

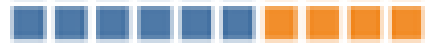

$\square \square \square \square \square \square \square \square \square$

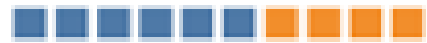

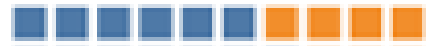

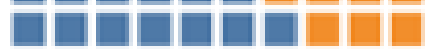

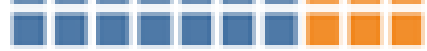

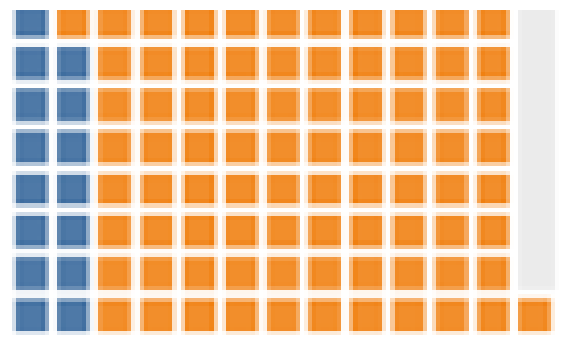

FALSE

TRUE

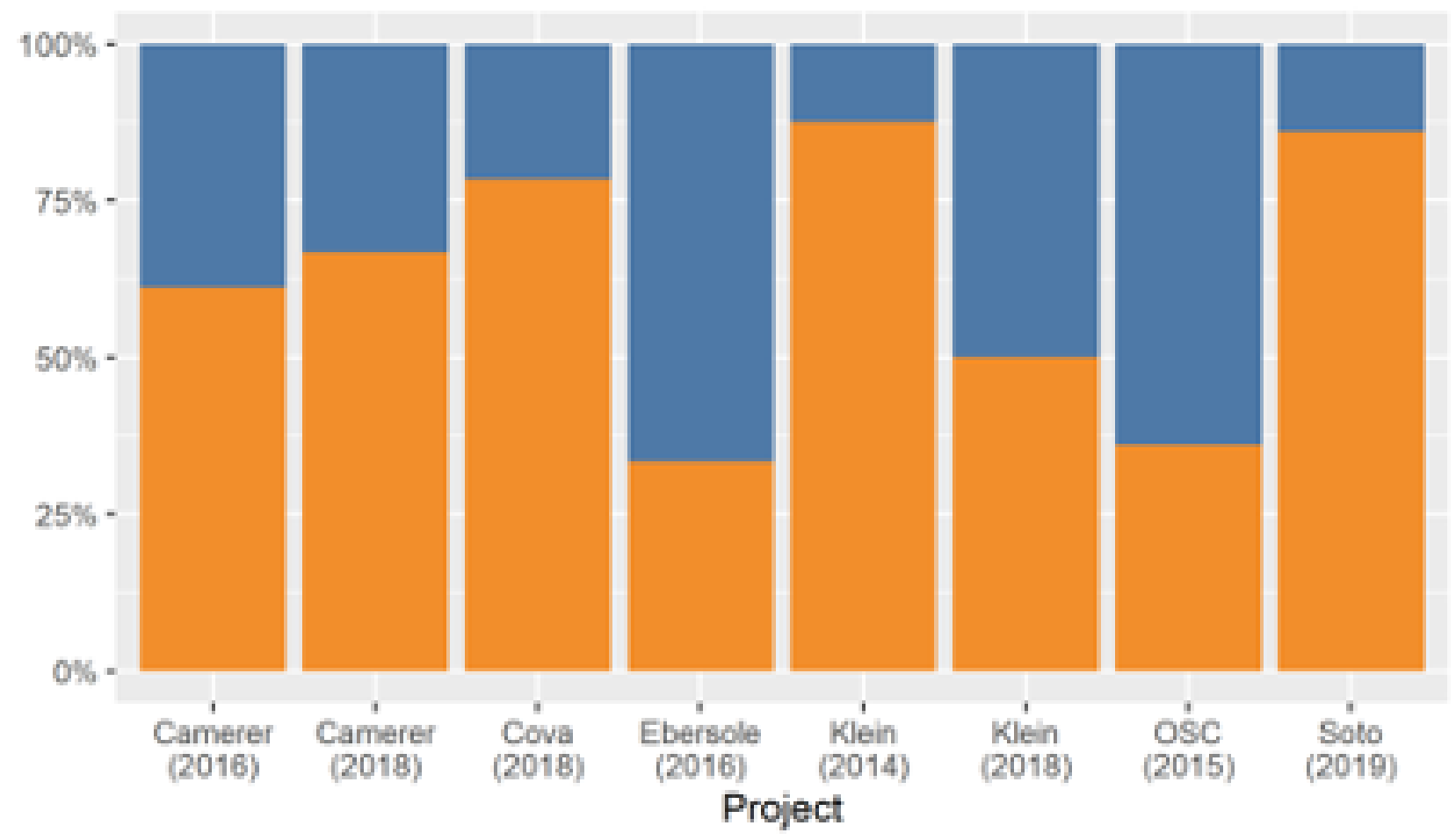

Replication significant in the original direction

\section{Claim Characteristics Database}


(Alternative name - "Correlates of Replicability Database")

\section{VARIABLE SELECTION}

Explain process for choosing correlates of replicability.

The final subset of variables chosen for collection was constrained by what we could feasibly manually code, and what we might be able to programmatically scrape.

\section{MANUAL CODING (ELLIOT)}

- check this against the reported / analysed set in the data!

- Add metadata file I created for Fiona to send to Charles (and double check hashed out coder ID)

- Add coding book to repo https://docs.google.com/document/d/19II9GH1mqeAYrWWYa3cukujSpCIpQ8CP8I5mYu-pPck/edit\#

A team of 13 people manually coded each of the original studies using a standardised code book (Supp. Mat. A3 ). They extracted the sample size of the original study, the reported $p$ value in the original paper, and identified the type of statistical test performed. They also recorded whether an effect size was reported in the original paper, whether confidence intervals were reported around the original effect, whether the effect was an interaction or a main effect, whether the original data was available, whether materials were available, whether sample size planning was reported (e.g., a power analysis), whether the paper mentioned any sort of blinding, what the original population was (e.g., undergraduate college students) and whether any scale reliability analyses were reported for each of the papers.

\section{SCRAPER (BEC)}

We constructed an automated scraper to obtain p-values, sample size, and other correlates of replicability from the main text of published journal articles within this database.

\section{STATCHECK DATA (ELLIOT)}

\section{STATCHECK DATA}

- (note that this is a different process to the RepliCATS claims processing workflow)

- TODO ref for ESExtractor

- TODO insert code for this process (from the other repo), do not run due to CRAN computational constraints, but include for transparency and completeness 
processed using ESExtractor to separate columns of text, and then parsed through R Statcheck . Statcheck extracts statistical results in APA format from strings of text, recomputes the P-values, and compares this against the reported $\mathrm{P}$-value. For each article it computes the number of statcheck errors, where the reported $\mathrm{P}$ value is incongruent with the recomputed $\mathrm{P}$ value, and whether a decision error has been made, i.e. the reported result is significant, yet the recomputed result is not, or vice versa. Statcheck also accounts for errors and decision errors that can be explained by one sided-ness. Because statcheck can only detect errors in APA formatted statistical test results, we treat any papers that did not have at least one statcheck result (i.e., one check-able APA formatted statistical test) as missing data and exclude from all relevant analyses.

\section{- explain treatment of missing statcheck variables}

This returned at least one detected $\mathrm{p}$ value for 0 articles, at least one APA formatted test statistic for 107 of the 244 papers for which PDFs could be found (1 original article could not be retrieved). A total of 0 papers had at least one inconsistency NaN\% of the total sample (similar to the to $49.6 \%$ which were found in the original Statcheck paper; ). A total of 0 papers had at least one "gross inconsistency," where a correctly calculated $p$ value would be on the opposite side of .05 if correctly calculated, $\mathrm{NaN} \%$ of studies for which at least one statistical test result was extracted (similar to the $12.9 \%$ in ).

\section{Statchecking inferential tests of replications}

- $\quad$ explain this process- i.e. what is the difference between parsing through statcheck above, and checking the inferential test statistics

In addition, using manually extracted test statistics, degrees of freedom and $\mathrm{p}$ values, we parsed a total of 0 of the replicated test statistics through Statcheck (i.e., all studies for which these values were reported in full for the statistical test of interest, and which used $F, t$, chi square tests, or correlations).

Out of the 0 test statistics that could be checked, a total of 0 returned statcheck errors. Just 0 out of 0 of these studies failed to replicate according to the "statistically significant replication effect in the same direction as the original" criterion. Both of the studies that showed non-significant replication results were "decision errors" (i.e., errors in which the correctly calculated $p$ value was above .05 whereas the reported value was below .05). Because of the extremely low number of errors detected, we do not use this variable in any of the predictive models presented below.

\section{METADATA FROM WEB OF SCIENCE (ELLIOT)}

Additional metadata was downloaded for 0 of the 220 original articles. The 220 original articles which were not indexed by Web of Science are excluded from any analyses including citation counts, but areas of research were manually inserted. Four articles replicated as part of the experimental philosophy replication project which were classified as being in the area of research "Philosophy," and the rest which were replicated as part of psychology replication projects were classified as being from the area of research "Psychology." 


\section{Dataset Creation - Human assessments of replicability}

(RepliCATS known outcomes data, SIPS workshop data).

\section{Model Specification}

\section{Predictive Model (Elliot)}

Our full model is described by:

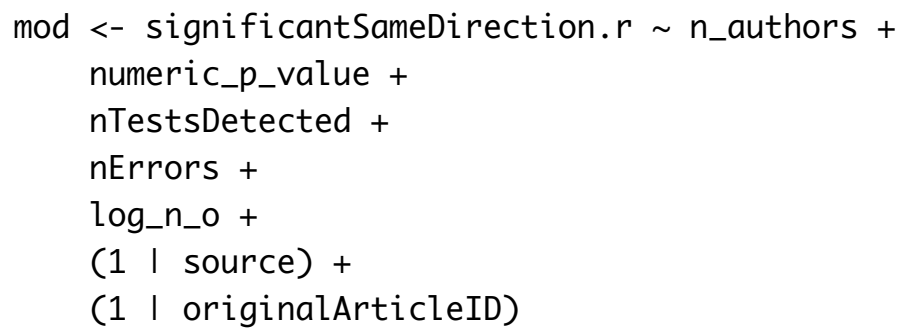

However, not all variables could be reliably scraped in our hold out prediction dataset. Consequently, we fitted combinatorial variations of the the full model for every claim, and selected only the model with the greatest number of terms to generate predictions per claim.

In all we specified 8 different models:

mod1 <- significantSameDirection. $r \sim n_{-}$authors + (1 I source $)+(1$ l originalArticleID $)$

mod2 <- significantSameDirection. $r \sim$ n_authors + p_value_original + (1 l source) + (1 l orig mod3 <- significantSameDirection.r $\sim$ n_authors + log_n_o + (1 I source) + (1 l originalArtic $\bmod 4<-$ significantSameDirection. $r \sim$ n_authors + p_value_original + log_n_o + (1 l source) + mod5 <- significantSameDirection. $r$ n_authors + nTestsDetected + nErrors + (1 I source $)+($ mod6 <- significantSameDirection. $r \sim$ n_authors + p_value_original + nTestsDetected + nErrors $\bmod 7$ <- significantSameDirection. $r \sim$ n_authors + nTestsDetected + nErrors + log_n_o + (1 I s mod8 <- significantSameDirection.r $\sim$ n_authors + p_value_original + nTestsDetected + nErrors

Formula:

significantSameDirection.r $\sim$ n_authors + numeric_p_value + nTestsDetected + nErrors + log_n_o + (1 I source) + (1 I originalArticleID)

Data: training_data

AIC BIC logLik df.resid

$153.93633177 .29618-68.96817$

Random-effects (co)variances: 
conaztzorial model:

$\begin{array}{lll}\text { Groups } & \text { Name } & \text { Std.Dev. } \\ \text { source } & \text { (Intercept) } & 0.9955 \\ \text { originalArticleID } & \text { (Intercept) } & 0.4946\end{array}$

Number of obs: 137 / Conditional model: source, 8; originalArticleID, 104

Fixed Effects:

Conditional model:

$\begin{array}{rrrr}\text { (Intercept) } & \text { n_authors } & \text { numeric_p_value } & \text { nTestsDetected } \\ 0.900374 & -0.490317 & -22.866478 & 0.003414 \\ \text { nErrors } & \text { log_n_o } & & \\ -0.861356 & 0.218999 & & \end{array}$

Like all frequentest multilevel modelling, this analysis assumes that the random effects for replication projects are normally distributed around an overall population parameter, implicitly conceptualizing each replication project as a random sample of possible replication projects.

The number of replication projects is quite low so estimates of the heterogeneity amount projects are somewhat imprecise, and the assumption that the random effects are normally distributed may not be reasonable for all variables. However, we believe that this approach is appropriate in balancing interpretability while acknowledging that these relationships will likely differ among replication projects.

\section{BayTriVar \& BayPriorsAgg (David)}

\section{Model Evaluation}

\section{Predictive Model(s) (Elliot)}

Training Dataset: Large-scale Replication Project Data ( 400 observations)

Full model, or Missingness on 25 known claims (provide naniar figure illustrating missingness, and distribution of models that were applied).

\section{Bayesian Models (David)}

Evaluation datasets:

- evaluate on hold out dataset (calibration validation) 
Evaluation metrics:

We employ 3 different metrics to evaluate the performance of both the predictive model and the Bayesian model:

- $\mathrm{AUC}$

- Brier Score

- and Classification Accuracy.

We calculate these measures using the aggreCAT: :confidence_score_evaluation() from the aggreCAT package.

\section{Results}

\section{Predictive Model Response Plots}

\section{$3 \times$ Model Eval \& Comparison (David)}

\section{Discussion}

\section{References}

\section{Supplementary material}

\section{SM1}

\section{ESTIMATING THE REPRODUCIBILITY OF PSYCHOLOGICAL SCIENCE (RPP; OPEN SCIENCE COLLABORATION, 2015)}

performed a total of 100 replications. They attempted to obtain a quasi-random sample by sampling from articles published in 2008 from the journals Psychological Science, Journal of Personality and Social Psychology, and Journal of Experimental Psychology: Learning, Memory, and Cognition.

Replication teams were instructed to select from a subset of the sampling frame (initially just the first 20 published articles from each journal were available, a pool that was expanded as it became difficult 
to match teams with articles). Project coordinators also actively recruited replication teams from the community that they thought had expertise to assess particular claims. Of the 488 articles that were published in the applicable journal issues, 158 (32\%) were eligible for selection. A total of 84 of the 100 replications conducted were of the final experiment in each original article. Sixteen replications deviated from this rule and used a different experiment due to a recommendation from the original authors or on the basis of feasibility. The statistical test that was chosen to reflect the replication

outcome was presented to the original authors as part of the design protocol, and was changed at the request of the original authors.

Open Science Collaboration (2015) reports that 47 articles were eligible for replication and were not claimed. Six of these studies were deemed infeasible due to practical constraints such as cost, historical dependence, or difficult to recruit samples, and were not presented to potential replicators. The remaining 41 were presented to potential replicators, but were not claimed. Open Science Collaboration (2015) reports that these projects "often" required access to rare populations, were likely to be expensive, required specialized materials (e.g., fMRI studies), or access to specialized knowledge.

\section{Participants}

Each replication study was powered on the basis of the original study's observed effect size. The average power to detect the original effect of the 97 studies with significant results was .92. Individual studies had sample sizes (at the individual level) of between min ( studyNs, na.rm $=\mathrm{T}$ ) and $\max ($ studyNs, na.rm $=\mathrm{T})($ mean $n=\operatorname{mean}($ studyNs, $n a . r m=T)$, median $=$ median ( studyNs, na.rm $=\mathrm{T}), \mathrm{sd}=\mathrm{sd}($ studyNs $))$.

\section{Extraction and inclusion rules}

Three original studies which did not report that their findings were indicative of a non-zero effect were excluded from those studies extracted from . Three studies for which $\mathrm{z}$ transformed correlation coefficients could not be extracted due to missing data in the downloaded data set were also excluded from analysis (these included 1 study which used multiple statistical tests in the original and replication studies, one study in which the replication and original study used different statistical tests, and one study for which the effect size was reported as a beta coefficients without test statistics or degrees of freedom). Effect sizes for original and replication studies are included for nrow (data) out of 97 studies replicated studies from which reported having found a non-zero effect.

\section{Sampling frame and reported deviations}

Camerer et al. (2018) replicated all 21 studies that were (a) published between 2010-2015, (b) in the 
hypothesis with a statistically significant finding," and that (e) "were performed on students or other accessible subject pools." In order to choose which of the original studies' statistical tests to use as the replication outcome, they selected the statistical tests that they subjectively identified as the most important in the first reported study that reported a significant treatment effect. When they could not identify a single most important effect they randomly selected from those that they identified as potential candidates. Camerer et al. (2018) report that there were no deviations from original studies' protocols for seven replications, "minor" deviations for 12 , and an "unintended" deviation for one study.

\section{Participants}

Camerer et al. (2018) took a two stage sampling procedure, where each study was initially replicated with a sample large enough to ensure $90 \%$ power to detect an effect $75 \%$ of the size of the original study's observed effect size. If there were no significant results in this first study, a second study was performed with $90 \%$ power to detect $50 \%$ of the original effect size. For one study, there was a significant result in the same direction as the original study in the first wave of replication data collection, but a second wave was still performed due to an error.

\section{Extraction and inclusion rules}

Original and replication studies were included for all allData $\%>$ filter (abrev == "natSci") $\therefore>$ nrow ( ) performed replications. For all cases in the Nature Science reproducibility project where multiple replication studies were performed for a single effect we performed a fixed effects metaanalysis using the metafor $\mathrm{R}$ package to estimate a meta-analytic effect size estimate. The effect size, standard errors and sample sizes used in the current study reflect this pooled estimate. This method leads to one study more "replicating" according to the 'statistical significance in the same direction of the original study' criterion than was originally reported in , where they used the $p$ value from the largest performed study instead of a pooled estimate.

\section{Sampling frame}

Soto (2019) extracted 78 trait-outcome associations (i.e., associations between The Big Five personality traits and life outcomes like volunteerism or politically conservatism) from a review article that presented a total of 86 associations between the Big Five traits and life outcomes. Soto and a research assistant extracted the empirical evidence behind these associations, and selected the 78 that were replicated on the basis of feasibility. In Soto (2019) the 78 trait-outcome associations were the primary units of analysis for estimating replicability, whereas in the current study we used the study level for comparability with the other replication projects. In total sum(allData\$abrev == "loopr" ) studies were included in the current analysis. 


\section{Deviations from original study protocols}

All replications were surveys administered using Qualtrics. The replications were all conducted in one of two large surveys. A single measure of the Big 5 personality traits was administered rather than the disparate versions used in each individual original study. The outcomes were assessed using measures that Soto reports were "selected to follow the original studies as closely as was feasible." Soto reports that most outcome measures were administered using the same measure, but some interview questions were altered to fit a survey format and some lengthy questions or measures were shortened. As this means that some replication studies used shorter form versions of the original data collection instruments, all significance tests or effect size analyses in the current paper use the effect sizes that Soto (2019) reports have been disattenuated using the Spearman-Brown prediction formula and Spearman disattenuation formula to estimate the trait-outcome associations that would be expected if the measures had used the same number of items as the original study (Lord \& Novick, 1968).

\section{Participants}

Two young adult (18-25 year olds) and two adult samples (18 years of age or older) were recruited using quota sampling. Quota sampling was employed in order to match the US population in terms of sex, race and ethnicity in both the adult and young adult samples, and additionally for age, educational attainment and household income in the adult samples. Samples of 1559 adults and 1550 young adults completed Survey 1 (including approximately half of the replication studies), and samples of 1512 adults and 1505 young adults completed Survey 2 (which included the remaining replication studies). Soto reports that this yielded a minimum power of $97.3 \%$ to detect a correlation of just .1 using a twotailed test with an alpha of .05.

\section{Extraction and inclusion rules}

We include a total of sum(allData\$abrev == "loopr" ) of the 121 trait-outcome associations in the current analysis. As some replication studies used shorter form versions of the original data collection instruments, all significance tests or effect size analyses use Soto (2019)'s disattenuated effect size estimates using the Spearman-Brown prediction formula and Spearman disattenuation formula to estimate the trait-outcome associations that would be expected if the measures had used the same number of items as the original study (Lord \& Novick, 1968). Effect sizes in Soto (2019) that were only reported as beta coefficients were not converted to Fisher z scores as not enough information was available in Soto's data set to do so. Effect sizes were extracted for original and replication studies for sum(! is.na(allData\$fis.o) \& ! is.na(allData\$fis.r) \& allData\$abrev == "loopr") out of sum(allData\$abrev == "loopr") included studies. Following the other large scale replication studies, the signs of the original and replication study effect sizes were inverted for analysis if the original effect was negative. 
provide evidence for the effects under test), these were removed from analysis. Effect sizes were reported by Cova et al. (2018) and are included in the current study for \# Number, original and replication studies, out of an original 37 replicated studies with significant original results. The four studies for which no effect sizes were reported performed analyses for which Cova et al. (2018) could not develop reasonable effect size estimates (e.g., a Sobel test, GEE analysis).

\section{Sampling frame and deviations from original protocols}

Cova et al. (2018) sampled their replication targets from a list of experimental philosophy papers hosted by Yale's University's Experimental Philosophy Lab (http://experimentalphilosophy.yale.edu/ExperimentalPhilosophy.html), and filtered these down to articles published in one of 35 scientific journals that Cova and Strickland judged would likely have published experimental philosophy research. From the remaining articles, they sampled three papers per year for the years 2003 to 2015, selecting the most cited paper (according to Google scholar) and two random studies (barring the year 2003 where there were only two applicable papers). This left them with a list of 38 studies which were assessed for feasibility. They report that four studies were identified as being potentially difficult to replicate (as they required access to special populations, three papers requiring access to non-English speakers and one requiring participants with high-functioning autism). In order to ensure that they achieved at least their goal number of studies, they sampled an additional replacement for each of the original studies they were concerned might be practically infeasible

(either picking the second most cited article of the year if the potentially infeasible study was selected as it was the most cited, or an additional random article), but continued to attempt to replicate all studies. In the end, two of the studies that were identified as being potentially infeasible were replicated, and two were not. The "more feasible" replication studies were also included in the final analysis if they were finished by the end of the project's duration. This left a total of 40 completed replications, 12 replications of most the most cited article of the years in the sampling frame, two replications of the second most cited article in a given year, and 26 studies which were randomly chosen from each year's set of experimental philosophy studies.

Cova et al. (2018) report that there was a major deviation in protocol for one study, where text was presented on screen for a set duration instead of being removed after participants responded, which they suggest may have reduced participants' incentive to quickly respond.

\section{Participants}

The research teams were based in Brazil, France, Lithuania, Netherlands, Spain, Switzerland, United Kingdom and the United States. Most studies were replicated using online samples (35 studies) and four used university students, unlike the original studies of which 25 used collage samples, 10 used online samples, and four involved local pedestrians. Cova et al. (2018) report that their average replication sample size was 206.3 , sd $=131.8$. For the 32 studies reporting a significant result for which they could perform a power analysis, the average level of statistical power to detect the original effect size was 0.88 (SD =0.14), 26 had a power $>0.80$ to detect the original sample size, and 18 had a power $>.95$. 


\section{Extraction and inclusion rules}

included three replications of original studies which were non-significant (and which did not claim to provide evidence for the effects under test), these were removed from this analysis. Effect sizes were reported by Cova et al. (2018) and are included in the current study for sum(! is . na (covdat $\$$ fis.o) \& ! is.na(covdat\$fis.r) \& ! is.na(covdat\$n.o) \& ! is.na(covdat\$n.r)), original and replication studies, out of an original 37 replicated studies with significant original results. The four studies which are not included did not report usable effect sizes, or information from which standardised effect size estimates could be developed.

\section{Many labs 1}

\section{Sampling frame}

Many Labs 1 replicated a non-random sample of 13 effects from 12 original articles. They report that the replicated studies were chosen on the basis of being (a) suitable for online presentation, (b) able to be presented "quickly" and (c) to have a simple, two condition design (except for a single a correlational study which was included). The replication materials were translated into the local language of each sample.

\section{Participants}

Klein et al. (2014) replicated each effect across 36 samples and settings ( 27 laboratory, 9 online, and 25 in the USA and 11 in non-US locations) including a total of 6344 participants. The individual studies each included between 4896 and 6336 participants.

\section{Extraction and inclusion rules}

One of the original studies in Many labs 1 did not report an effect size or test statistic so is not included in the current sample. No effect size was extractable for one original study. Four different operationalisations of anchoring effects were tested, all of which are included in the current analysis, leading to a total of 15 replication studies being included from this project.

\section{MANY LABS 2}

\section{Sampling frame}

Many labs 2 replicated a non-random sample of 28 effects. In order to select the claims that were replicated, they (a) held an open nomination round that fit a set of desired characteristics, elicited ideas within the project team, and asked "independent experts" who worked in the field of 

can be implemented via a web browser, (b) not have more than two conditions in an experimental design, (c) have one outcome (dependent variable), (d) be correlational or experimental designs, though the latter is preferred, and (e) examine a psychological topic." There were also a set of desired characteristics, that the study should: "(a) be"important" in at least one of the many variety of ways of demonstrating importance, (b) be brief, (c) be a direct replication of an existing published design from which an original effect size can be determined)" (see https://osf.io/uazdm/ for the coordinating proposal).

After over 100 claims were nominated, the Klein et al., chose the replicated claims on the basis of the claims being (a) suitable for online presentation, (b) able to be presented quickly, (c) citation count (more being judged more positively), (d) to have a simple, two condition design or, at a lower priority, a correlational design, (e) to have a high level of "general interest," and ( $f$ ) to be applicable to a general sample of adults. Secondarily, Klein et al., report that they aimed to ensure a diversity of claims, including (a) claims that had both limited and extensive literatures across samples and settings, (b) effects for which it was expected that there would be high and low effect size heterogeneity, (c) "classic" and "contemporary" effects, (d) effects which cover a broad range of subareas within social and cognitive psychology, (e) effects from a diversity of research teams, and finally (f), effects from a variety of journals.

For all chosen claims, the original study's corresponding author were contacted (if possible) and asked for the original materials, and asked if there were any limitations or moderators that the replication teams should be aware of before attempting the replication. Klein et al., report that this process eliminated some studies, at which point the effect was eliminated from consideration and replaced with another of the nominated claim or the original study's authors were asked to help identify alternative studies for replication. In one case, the original authors expressed "strong concerns" about the study being included in this project, and this claim was removed.

After this process, 31 effects remained, which were split into two 30 minutes "slates," and pilot tested. Three effects were removed for length, and some studies were shortened in order to fit into the two 30 minutes slates after pilot testing. This left 28 effects that were replicated.

\section{Participants}

Labs were invited to participate in an open call made in 2014. The only criteria for inclusion was that a lab had to agree to include at least 80 participants. A total of 125 samples were recruited in this study. The total sample size was 15305 , with ns of at least min (studyNs) in each replication study after exclusions (mean $n=\operatorname{mean}($ studyNs $), s d=\operatorname{sd}($ studyNs $), \operatorname{median}=\operatorname{median}($ studyNs $)$ )

\section{Extraction and inclusion rules}

A total of 22 of 28 paired original and replication studies are included in the above analyses. Six studies from were removed because the original and replication studies examined a difference in effect sizes seen in different conditions, and the effects were not directly tested against each other in the original papers. 


\section{MANY LABS 3}

\section{Sampling frame}

A non-random sample of nine original studies were replicated, and one conceptual replication was performed. The conceptual replication was excluded from the current analyses. Ebersole et al., report that they aimed to identify two-condition experiments or correlational designs. Seven out of the nine included studies were included in a single computer delivered experiment, and were followed by extensive individual difference and data quality indicators. Two experiments were exclusively performed in person as, in one case, the study required participants to physically hold a clipboard, and in one as the original authors said that the experiment needed to be administered using a paper and pencil format.

\section{Participants}

The replication included 20 university participant pools at universities in the United states and Canada $(\mathrm{N}=2,696)$ and an mTurk online sample $(\mathrm{N}=737)$. Individual studies had ns of at least min ( $\mathrm{studyNs}$ ) after exclusions (mean $n=\operatorname{mean}($ studyNs), $s d=s d($ studyNs $)$, $\operatorname{median}=\operatorname{median}($ studyNs $)$ )

\section{Extraction and inclusion rules}

Original and replication effect sizes were extracted for all 9 original and replication studies from , excluding a study they term a "conceptual replication."

\section{ECONOMICS REPLICATION PROJECT}

\section{Sampling frame and deviations}

Camerer et al. (2016) replicated all 18 between-subject laboratory experimental papers published in the American Economic Review and the Quarterly Journal of Economics between 2011 and 2014. For 17 of 18 studies, the same software was used to conduct the replications as was used in the original experiment. In one study the software used was different as the original program was no longer available.

\section{Participants}

Two American teams, one Swedish, and one Austrian replication team conducted four or five replications each. Camerer (2016) reports that the replication sample sizes were chosen to ensure that each replication study had at least $90 \%$ power to detect the original study's effect size using the original analysis strategy. Individual studies had ns of at least min ( studyNs) (mean $n=$ 


\section{Extraction and inclusion rules}

The economics replication project . Original and replication effect sizes for all sum(allData\$abrev $==$ ' econ' ) studies were reported in correlation coefficients and all are included in this analysis.

\section{SM2}

\section{EFFECT SIZE CONVERSIONS}

All statistical tests extracted were transformed into correlation coefficients as follows, using the methods reported in .

t statistics:

$r=\sqrt{\frac{t_{o b s}^{2} \times(1 / d f)}{\left(t_{o b s}^{2} / d f\right)+1}}$

Where $t_{o b s}$ is the observed t statistic and $d f$ is the degrees of freedom of the test.

F statistics:

$r=\sqrt{\frac{F_{o b s} \times\left(d f_{1} / d f_{2}\right)}{F_{o b s} \times\left(d f_{1} / d f_{2}\right)+1}} \times \sqrt{\frac{1}{d f_{1}}}$

Where $F_{o b s}$ is the observed $\mathrm{F}$ statistic and $d f_{1}$ is the degrees of freedom of the numerator and $d f_{2}$ is degrees of freedom of the denominator.

Chi square statistics:

$r=\sqrt{\frac{\chi_{o b s}^{2}}{d f+2}}$

Where $\chi_{o b s}^{2}$ is the observed $\chi_{o b s}^{2}$ statistic and $d f$ is the associated degrees of freedom.

All values were then transformed into fisher $\mathrm{Z}$ transformed correlation coefficients using:

$z=\frac{1}{2} \times \ln \left(\frac{1+r}{1-r}\right)$ 
